\title{
Erratum to: Brachistochrone with limited reaction of constraint in an arbitrary force field
}

\author{
Slaviša Šalinić • Aleksandar Obradović • \\ Zoran Mitrović • Srdjan Rusov
}

(C) Springer Science+Business Media B.V. 2012

\section{Erratum to: Nonlinear Dyn (2012) 69:211-222 DOI 10.1007/s11071-011-0258-1}

In the original publication, Eq. (15) should read as following:

$$
\begin{aligned}
& \frac{d \lambda_{y}}{d x}=-\frac{\partial H}{\partial y}=-\lambda_{v} \frac{\partial \Psi_{v}}{\partial y}+v \frac{\partial N_{n}}{\partial y}, \\
& \frac{d \lambda_{p}}{d x}=-\frac{\partial H}{\partial p}=\frac{\partial \Psi}{\partial p}-\lambda_{y}-\lambda_{v} \frac{\partial \Psi_{v}}{\partial p}+v \frac{\partial N_{n}}{\partial p}, \\
& \frac{d \lambda_{v}}{d x}=-\frac{\partial H}{\partial v}=\frac{\partial \Psi}{\partial v}-\lambda_{v} \frac{\partial \Psi_{v}}{\partial v}+v \frac{\partial N_{n}}{\partial v},
\end{aligned}
$$

where for $N^{\star}<N_{n}<N^{\star \star}$ it is $v \equiv 0$ and $v=$ $\lambda_{p} /\left(\partial N_{n} / \partial u\right)$ otherwise (see e.g. [1]).

The online version of the original article can be found under doi:10.1007/s11071-011-0258-1.

S. Šalinić $(\bowtie)$

Faculty of Mechanical Engineering, University of Kragujevac, Dositejeva 19, 36000 Kraljevo, Serbia e-mail: salinic.s@ptt.rs

S. Šalinić

e-mail: salinic.s@mfkv.kg.ac.rs

A. Obradović · Z. Mitrović

Faculty of Mechanical Engineering, University of Belgrade, Kraljice Marije 16, 11120 Belgrade 35, Serbia

S. Rusov

Faculty of Transport and Traffic Engineering, University of Belgrade, Vojvode Stepe 305, 11000 Belgrade, Serbia
This correction implies the following corrections in Sect. 4.

\section{Section 4.1}

The part " $\ell_{0}$ is the free length of spring" bellow Eq. (28) should read: $\ell_{0}$ is the length of the part above $x$-axis of the free length of the spring.

The values of the parameters $p_{f}$ and $x_{1}$ bellow Eq. (33) should be: $p_{f}=0.6912$ and $x_{1}=2.5203 \mathrm{~m}$.

The numerical calculations are done for $\ell_{0}=4 \mathrm{~m}$.

Corrected values in Table 1.

\section{Section 4.2}

Bellow Eq. (36), the following new sentence should be added:

Now, the multiplier $v$ is determined by (see [1]):

$v=\frac{\lambda_{p}+\lambda_{v} \frac{\partial \Psi_{v}}{\partial u}}{\frac{\partial N_{n}}{\partial u}}$.

Table 1 Numerical values of the parameters of brachistochrone curves for various values of the coefficient of viscous friction $k$

\begin{tabular}{llll}
\hline$k[\mathrm{~kg} / \mathrm{m}]$ & $p_{f}$ & $v_{f}[\mathrm{~m} / \mathrm{s}]$ & $x_{1}[\mathrm{~m}]$ \\
\hline 0.6 & 0.706629 & 3.47916 & 2.71499 \\
0.8 & 0.723655 & 3.21593 & 2.93336 \\
0.9 & 0.732851 & 3.0908 & 3.05211 \\
1.0 & 0.742551 & 2.96975 & 3.14159 \\
\hline
\end{tabular}


The four numerical steps in Sect. 4.2 should be replaced by the following three ones:

- Applying the Runge-Kutta method one solves in the interval $\left[0, x_{2}\right]$ a Cauchy problem of the system of differential equations (9) and (15), with the initial conditions $y(0)=0, p(0)=p_{f}, v(0)=v_{f}$, $\lambda_{v}(0)=0, \lambda_{p}(0)=0, \lambda_{y}(0)=\lambda_{y}$, where the nonsingular control (17) was obtained based on boundary value $N^{\star}=18 \mathrm{~N}$ of the constraint reaction. Now, applying the conditions (37) at the point $x_{2}$, numerical dependencies $f_{1}\left(p_{f}, v_{f}, x_{2}, \lambda_{y}\right)=0$ and $f_{2}\left(p_{f}, v_{f}, x_{2}, \lambda_{y}\right)=0$ are established.

- The differential equations (24) are integrated in the singular interval $\left[x_{2}, x_{1}\right]$. The values $y\left(x_{2}\right), p\left(x_{2}\right)$, $v\left(x_{2}\right), \lambda_{v}\left(x_{2}\right)$ obtained in the first step are taken for initial values, where a singular control was determined from the conditions (20).

- The final step is the integration of the differential equations (9) and (15) in the nonsingular interval $\left[x_{1}, x_{0}\right]$, the values $y\left(x_{1}\right), p\left(x_{1}\right), v\left(x_{1}\right), \lambda_{v}\left(x_{1}\right)$ obtained in second step as well as $\lambda_{p}\left(x_{1}\right)$ and $\lambda_{y}\left(x_{1}\right)$ determined according to (37), being taken for initial values, where the nonsingular control (17) was obtained on the basis of the boundary value $N^{\star \star}=0$ of the constraint reaction. Thus, numerical dependencies $y\left(x_{0}\right)=f_{3}\left(p_{f}, v_{f}, x_{1}, x_{2}, \lambda_{y}\right), v\left(x_{0}\right)=$ $f_{4}\left(p_{f}, v_{f}, x_{1}, x_{2}, \lambda_{y}\right)$, and $\lambda_{p}\left(x_{0}\right)=f_{5}\left(p_{f}, v_{f}, x_{1}\right.$, $\left.x_{2}, \lambda_{y}\right)$ are established.

Equation (38) should read:

$$
\begin{aligned}
& 0=f_{1}\left(p_{f}, v_{f}, x_{2}, \lambda_{y}\right), \\
& 0=f_{2}\left(p_{f}, v_{f}, x_{2}, \lambda_{y}\right), \\
& y\left(x_{0}\right)=f_{3}\left(p_{f}, v_{f}, x_{1}, x_{2}, \lambda_{y}\right), \\
& v\left(x_{0}\right)=f_{4}\left(p_{f}, v_{f}, x_{1}, x_{2}, \lambda_{y}\right), \\
& \lambda_{p}\left(x_{0}\right)=f_{5}\left(p_{f}, v_{f}, x_{1}, x_{2}, \lambda_{y}\right) .
\end{aligned}
$$

The first sentence bellow Eq. (38) should be replaced with:
Table 2 Numerical values of the parameters of brachistochrone curves for various values of speed $v_{0}$ (three-segment brachistochrone)

\begin{tabular}{llllll}
\hline$v_{0}[\mathrm{~m} / \mathrm{s}]$ & $x_{1}[\mathrm{~m}]$ & $x_{2}[\mathrm{~m}]$ & $p_{f}$ & $v_{f}[\mathrm{~m} / \mathrm{s}]$ & $\lambda_{y}[\mathrm{~s} / \mathrm{m}]$ \\
\hline 5 & 2.41248 & 0.585324 & 0.318344 & 7.42511 & 0.027754 \\
5.5 & 2.30418 & 0.42243 & 0.357016 & 7.79394 & 0.030944 \\
6 & 2.19576 & 0.243516 & 0.392991 & 8.17732 & 0.033317 \\
6.5 & 2.08774 & 0.048703 & 0.426203 & 8.57323 & 0.035002 \\
\hline
\end{tabular}

Table 3 Numerical values of the parameters of brachistochrone curves for various values of speed $v_{0}$ (two-segment brachistochrone)

\begin{tabular}{llll}
\hline$v_{0}[\mathrm{~m} / \mathrm{s}]$ & $x_{1}[\mathrm{~m}]$ & $p_{f}$ & $v_{f}[\mathrm{~m} / \mathrm{s}]$ \\
\hline 8 & 1.76849 & 0.508874 & 9.81917 \\
10 & 1.35671 & 0.585823 & 11.5744 \\
14 & 0.573338 & 0.671996 & 15.2642 \\
16 & 0.195741 & 0.696597 & 17.1602 \\
17 & 0.0094198 & 0.706191 & 18.1164 \\
\hline
\end{tabular}

Incorporating the conditions (10) and (16) into (38) and solving the obtained system of nonlinear equations for unknowns $p_{f}, v_{f}, x_{1}, x_{2}, \lambda_{y}$, one obtains the following solution: $p_{f}=0.234, v_{f}=6.7412 \mathrm{~m} / \mathrm{s}$, $x_{1}=2.6254 \mathrm{~m}, x_{2}=0.8626 \mathrm{~m}, \lambda_{y}=0.0185 \mathrm{~s} / \mathrm{m}$.

Corrected numerical values are presented in Tables 2 and 3 .

The values $\left(v_{0}\right)_{c r 1}$ and $\left(v_{0}\right)_{c r 2}$ should be replaced with the following values: $\left(v_{0}\right)_{c r 1} \approx 6.61902 \mathrm{~m} / \mathrm{s}$ and $\left(v_{0}\right)_{c r 2} \approx 17.05076 \mathrm{~m} / \mathrm{s}$.

In the last sentence in Sect. 4.2, the part "is higher than" should be replaced with "is the same as".

Note that, as the changes of numerical parameters used to draw graphs in Figs. 3, 4, 8 and 9 are around $10^{-3}$ in the order of magnitude, the graphs mentioned do not have any visible changes in their form.

\section{References}

1. Leitman, G.: An Introduction to Optimal Control. McGrawHill, New York (1966) 